\title{
Pattern of Syringomyelia in Presumed Idiopathic and Congenital Scoliosis
}

\author{
Simanchal Prosad Mohanty, Madhava Pai Kanhangad, Sibin Saifuddin, Jayakrishnan K. Narayana Kurup \\ Department of Orthopaedics, Kasturba Medical College, Manipal Academy of Higher Education, Manipal, India
}

\section{Study Design: Retrospective study.}

Purpose: This study was undertaken to compare the patterns of syringomyelia in patients with presumed idiopathic and congenital scoliosis.

Overview of Literature: The incidence of neuraxial anomalies presenting as idiopathic scoliosis ranges from $2 \%$ to $14 \%$; the common ones are idiopathic syringomyelia (IS) and Chiari malformation type 1 (CM1) with syringomyelia. Some authors have speculated that scoliosis is caused by the asymmetrical compression of anterior horn cells by a syrinx, which causes an imbalance of the trunk musculature. In congenital scoliosis, syringomyelia is the second commonest cord anomaly, and the deformity progression depends upon the underlying vertebral abnormality, the location of the abnormality, and the age of patient, and is independent of the intraspinal anomaly.

Methods: We analyzed the radiological records of 44 consecutive patients with scoliosis and syringomyelia. Of these 44 patients, 13 had IS, 12 had CM1, and 19 had congenital scoliosis. The radiographs were evaluated to determine the curve magnitude, sagittal alignment, side of convexity, and type of vertebral anomaly, if any. T1- and T2-weighted magnetic resonance imaging of the whole spine was analyzed to determine the presence of craniovertebral anomalies, syrinx length, syrinx diameter, and syrinx-cord ratio (SCR). Results: The frequency of left convex curves was $26.1 \%$, with no significant differences across the three groups. The mean length of the syrinx was $7.2 \pm 4.9$ vertebral levels, and the mean SCR was $0.39 \pm 0.2$. The mean syrinx length was significantly higher in patients with CM1 and IS, compared to patients with congenital scoliosis. The mean SCR was highest in patients with CM1. In congenital scoliosis, syringomyelia was seen most frequently in patients with a failure of formation, and $63.2 \%$ had concomitant cord anomalies. Conclusions: Syrinxes were smaller in size and length in patients with congenital scoliosis, and are distinct from those seen in IS and CM1.

Keywords: Scoliosis; Syringomyelia; Chiari malformation; Magnetic resonance imaging

\section{Introduction}

Syringomyelia is characterized by the presence of a longitudinally oriented, fluid-filled cavity within the spinal cord, and it is labeled as "idiopathic" when no attributable cause is detected on magnetic resonance imaging (MRI). The true incidence of idiopathic syringomyelia (IS) is hard to ascertain, as many patients remain asymptomatic.

\footnotetext{
Received May 5, 2020; Revised Jul 27, 2020; Accepted Jul 27, 2020

Corresponding author: Madhava Pai Kanhangad

Division of Spine Surgery, Department of Orthopaedics, Kasturba Medical College, Manipal Academy of Higher Education, Manipal 576-104, Karnataka, India

Tel: +91-820-2922754, Fax: +91-820-2571934, E-mail: madhava.pai@manipal.edu, kmadhavapai@gmail.com
} 
The other common etiologies for syringomyelia are Chiari malformation type 1 (CM1), congenital scoliosis, trauma, tumors, and infection. The incidences of syringomyelia in CM1 and congenital scoliosis range from $60 \%$ to $85 \%$ and $6 \%$ to $30 \%$, respectively [1-4].

There is a close relationship between syringomyelia and scoliosis. The incidence of neuraxial anomalies presenting as idiopathic scoliosis ranges from $2 \%$ to $14 \%$; the common anomalies are IS and CM1 with syringomyelia. In the absence of vertebral anomalies, some authors have labeled these cases as presumed idiopathic scoliosis $[5,6]$. While the exact pathogenesis of scoliosis remains unclear, some authors have speculated that asymmetrical compression of anterior horn cells by a syrinx causes an imbalance of the trunk musculature, resulting in scoliosis [7-9]. The incidences of scoliosis in IS and CM1 with syringomyelia are about $14 \%-50 \%$ and $25 \%-85 \%$, respectively. However, the incidence of scoliosis in patients with CM1 without syringomyelia is about $4 \%$, which suggests the presence of alternate pathomechanisms [9-12]. Syringomyelia is the second commonest cord anomaly seen in congenital scoliosis. In congenital scoliosis, the progression of deformity is independent of the size and location of the syrinx, and instead depends on underlying vertebral anomalies. Since this is a developmental abnormality, the majority of patients with congenital scoliosis have concomitant cord anomalies [2-4,13].

While various studies have described the patterns of syringomyelia in presumed idiopathic and congenital scoliosis, few studies have compared them [14,15]. In the current study, we attempted to determine the patterns of syringomyelia and spinal deformity in patients with presumed idiopathic and congenital scoliosis. We also compared the curve patterns and MRI characteristics of syringomyelia in patients with CM1, IS, and congenital scoliosis.

\section{Materials and Methods}

In a retrospective study, we analyzed the radiographic records of 582 consecutive patients who presented between January 2010 and June 2018 with spontaneous-onset scoliosis before the age of 18 years. Standardized standing anteroposterior (AP) and lateral radiographs of the whole spine were obtained in all patients. All patients underwent an MRI of the spine to detect craniovertebral and cord anomalies. We excluded 538 patients whose MRIs showed no syringomyelia; of these, 296 patients were idiopathic and 208 had congenital scoliosis. Patients with histories of spinal trauma, infection, and tumors were also excluded. Thus, 13 patients with IS, 12 patients with CM1, and 19 patients with congenital scoliosis formed the basis of this study. In the current series, all patients with CM1 had syringomyelia, and none of the cases with CM1 had congenital vertebral anomalies.

\section{Radiological evaluation}

The digitalized radiographs were analyzed twice each by two independent observers in a blinded fashion, using a RadiAnt DICOM viewer ver. 4.6.5 (Medixant, Poznań, Poland). In cases with a difference in the two observers' readings, the decision of the senior author was considered to be final. The upper-end, lower-end, and apical vertebrae were noted on the standing AP radiographs. In patients with congenital scoliosis, the vertebral anomalies were classified according to the scale created by Hedequist and Emans [16]. The location and side of scoliosis was recorded, and the curve magnitude was measured using the method described by Cobb [17]. The sagittal alignment from T5 to T12 was determined using the modified Konstam's angle [18].

\section{Evaluation of intraspinal anomalies}

The T1- and T2-weighted MRIs of the whole spine, including the craniovertebral junction, were analyzed to detect the presence of syringomyelia, CM1, a split cord malformation (SCM), a tethered cord, a low-lying conus, or any intradural mass. In patients with syringomyelia, the T1- and T2-weighted sagittal and axial sections were analyzed for the location of the syrinx, length of the syrinx (as per the whole number of vertebral segments traversed in the sagittal sections), and AP diameter of the syrinx at the level of maximum expansion, which were noted on the axial sections (Fig. 1) [7]. On transverse images, the distances between the center of the syrinx and the bilateral rims of the spinal cord were measured at the upper and lower ends of the syrinx. The syrinx deviation rate (SDR) was calculated as described by Zhu et al. [8] (Fig. 2): (right-side distance at the upper end+right-side distance at the lower end)/(left-side distance at the upper end+leftside distance at the lower end). The syrinx was considered to be centrally located if the SDR was between 0.9 and 1.1, 

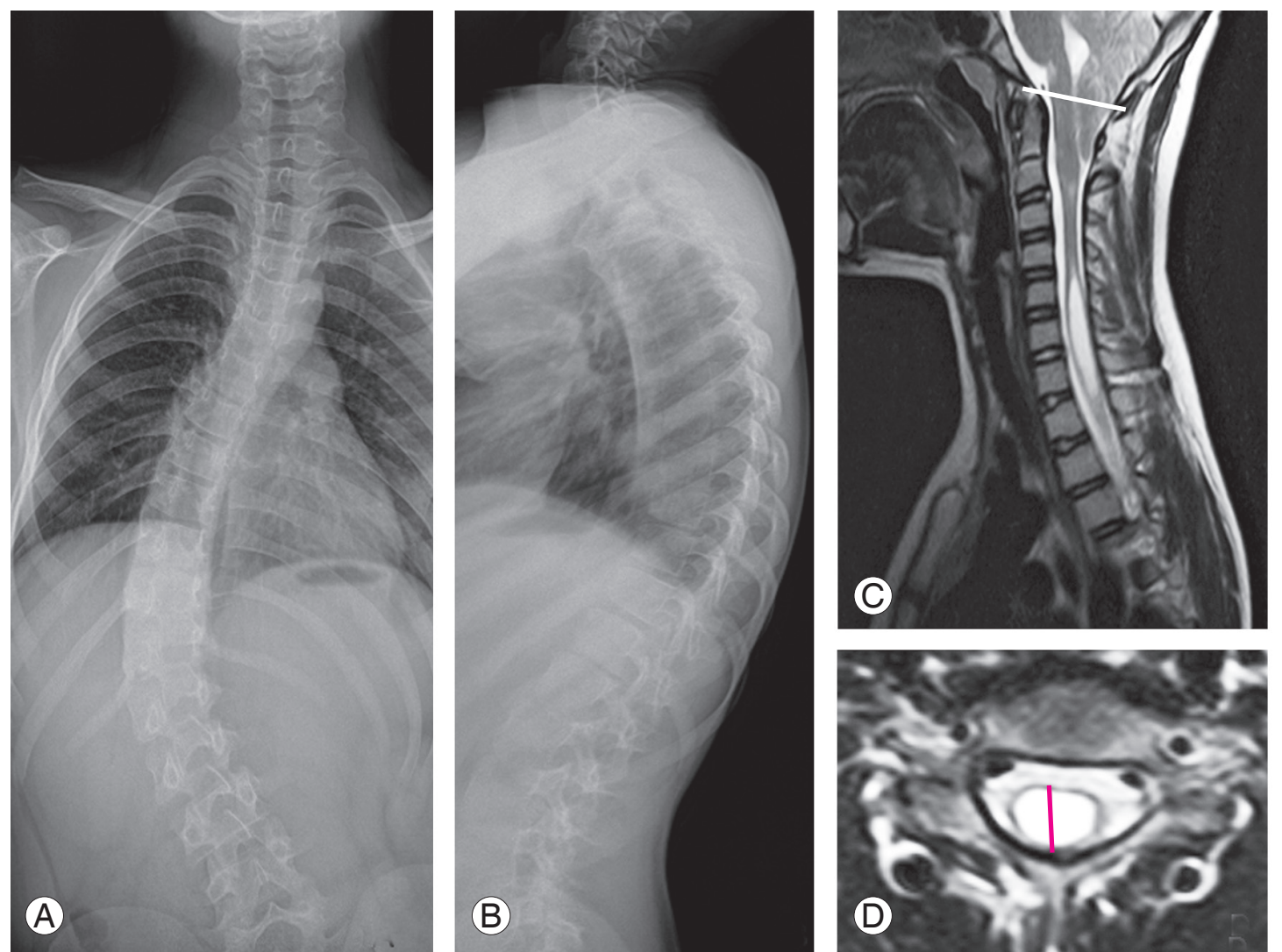

Fig. 1. A 14-year-old boy with presumed idiopathic scoliosis, whose anteroposterior (A) and lateral (B) radiographs showed a right thoracolumbar scoliosis. (C) Magnetic resonance imaging demonstrated presence of cerebellar tonsils $19 \mathrm{~mm}$ distal to the McRae's line and (D) diameter of the syrinx at the level of maximal expansion (red line).
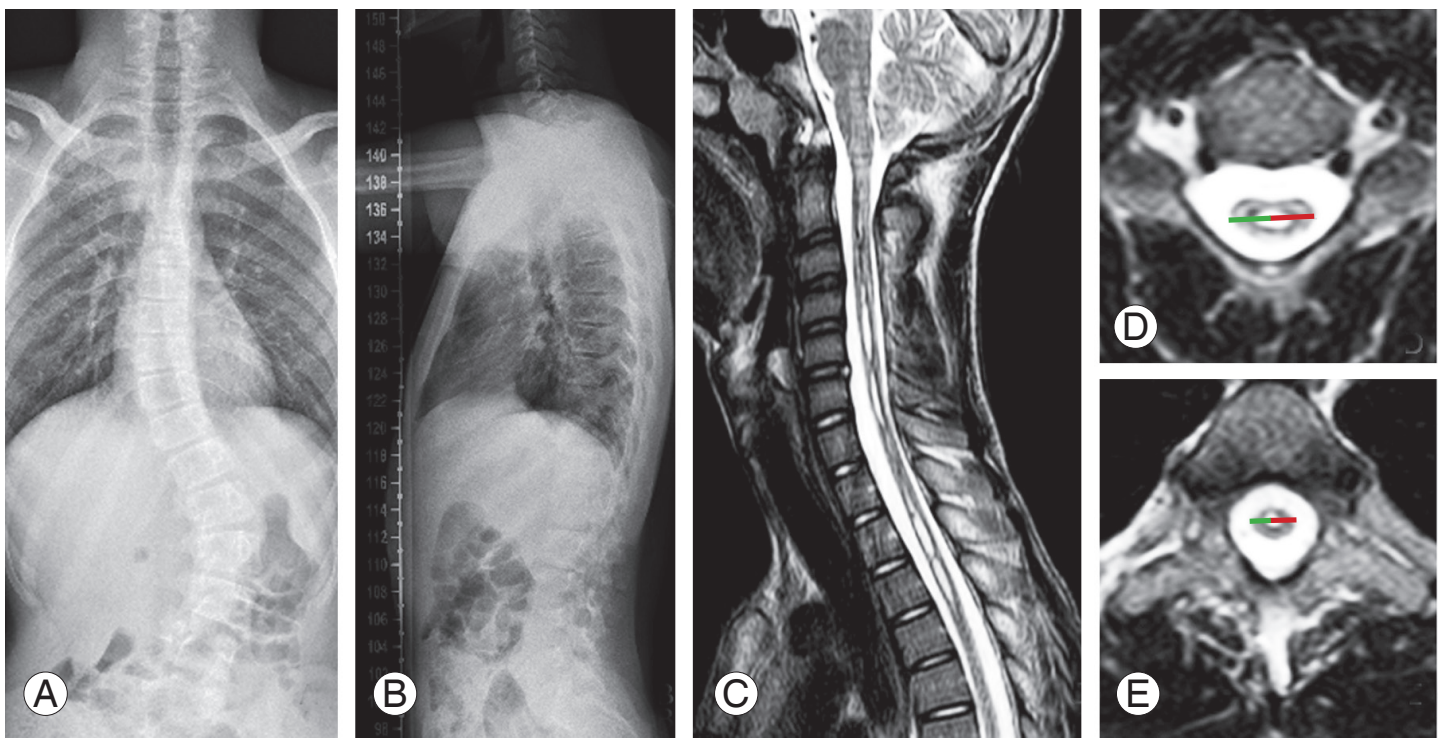

Fig. 2. A 13-year-old girl with presumed idiopathic scoliosis, whose anteroposterior (A) and lateral (B) radiographs showed a left lumbar curve. (C) Magnetic resonance imaging demonstrated slit like syrinx from C2-T3. Axial sections at the upper (D) and lower (E) end of the syrinx were analyzed to determine syrinx deviation rate (green \& red lines). 

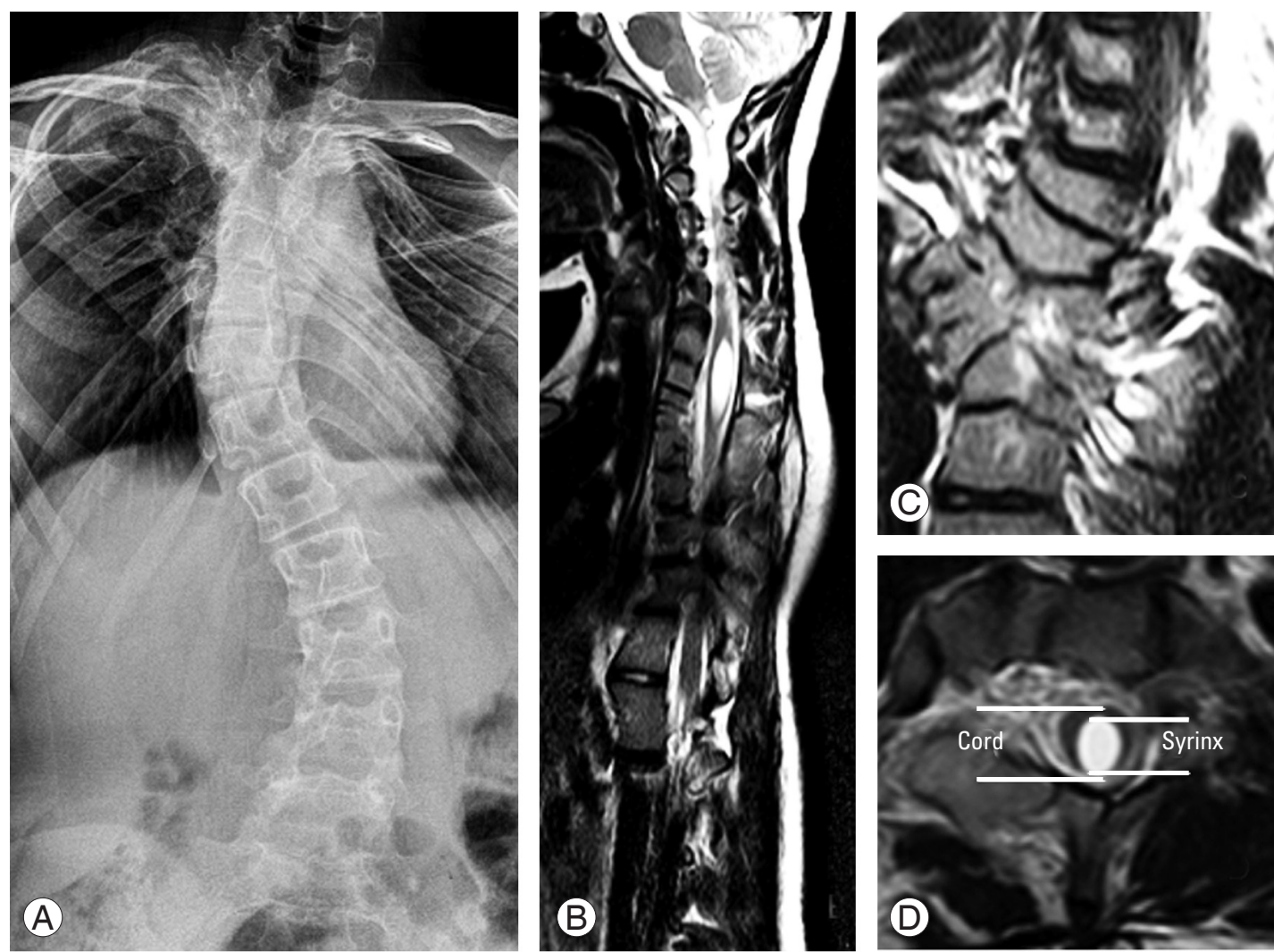

Fig. 3. A 10-year-old girl with congenital scoliosis, whose (A) anteroposterior radiographs showed mixed vertebral anomalies. (B) Magnetic resonance imaging showed syrinx in the thoracic spine, with mixed vertebral anomalies (C). (D) The syrinx-cord ratio was measured at the site of maximal expansion of the syrinx.

located to the right if the SDR was greater than 1.1, and located to the left if the SDR was $<0.9$. The syrinx-cord ratio (SCR) was calculated by dividing the maximal syrinx diameter by the diameter of the cord at the same level (Fig. 3). The syrinxes were classified as small (SCR, 0.2-0.25), medium (SCR, 0.25-0.5), and large (SCR >0.5) [7,19]. In the current study, CM1 was defined as the presence of a cerebellar tonsil at least $5 \mathrm{~mm}$ distal to McRae's line.

\section{Statistical analysis}

IBM SPSS for Windows ver. 20.0 (IBM Corp., Armonk, NY, USA) was used for the statistical analysis. The intraclass correlation coefficients were used to determine the inter- and intra-observer variability of the Cobb angle and SCR. Quantitative variables, such as the frequencies of left convex curves and vertebral and cord anomalies, were expressed as percentages. Continuous variables, such as age, Cobb angle, SCR, and length of syrinx, were expressed as means \pm standard deviations. A one-way repeated analysis of variance was used to compare age, Cobb angle at the time of presentation, and SCR. If a significant difference was noted between groups, a Student $t$-test was used for a pairwise comparison. The chi-square test was used to compare the frequencies of right and left convex curves and the male-to-female ratios between patients with CM1 with syringomyelia, IS, and congenital scoliosis with syringomyelia. A $p$-value of less than 0.05 was considered to be significant.

\section{Ethical considerations}

All procedures performed in studies involving human participants were in accordance with the ethical standards of the institutional and/or national research committee and with the 1964 Helsinki Declaration and its later amendments or comparable ethical standards. Ethical approval by the Institutional Review Board of Kasturba Medical College, Manipal Academy of Higher Education, Manipal was obtained prior to the start of the study (IRB 
Table 1. Descriptive statistics of studied sample

\begin{tabular}{lcccc} 
Characteristic & $\begin{array}{c}\text { Chiari malformation type 1 } \\
(\mathrm{n}=12)\end{array}$ & $\begin{array}{c}\text { Idiopathic syringomyelia } \\
(\mathrm{n}=13)\end{array}$ & $\begin{array}{c}\text { Syringomyelia in congenital scoliosis } \\
(\mathrm{n}=19)\end{array}$ & $\begin{array}{c}p \text {-value } \\
\text { Age at presentation }(\mathrm{yr})\end{array}$ \\
\hline $11.8 \pm 3.4$ & $14.8 \pm 2$ & $6: 4 \pm 1.9$ & 0.23 \\
\hline Male:female ratio & $3: 1$ & $49.8 \pm 9.7$ & $4: 12$ & 0.11 \\
\hline Cobb angle $\left(^{\circ}\right)$ & $55.4 \pm 10.2$ & $9: 4$ & $13: 6$ & $<0.05$ \\
\hline Right:left convex curve & $5: 1$ & $8(61.5)$ & $8(42.1)$ & 0.62 \\
\hline Thoracic hyper kyphosis & $9(75)$ & $7.5 \pm 4.1$ & $4.6 \pm 0.3$ & 0.18 \\
\hline Mean length of syrinx & $10.9 \pm 5$ & $0.38 \pm 0.3$ & $0.34 \pm 0.04$ & $<0.05$ \\
\hline Mean syrinx cord ratio & $0.51 \pm 0.1$ & & $<0.05$ &
\end{tabular}

Values are presented as mean \pm standard deviation, number, or number (\%).

approval no., ECR/146/Inst/KA/2013). In formed consent was obtained from all individual participants included in the study. This article does not contain any studies with animals performed by any of the authors.

\section{Results}

The intraclass correlation coefficient ranged from 0.81 to 0.85 , suggestive of good inter- and intra-observer agreeability. The descriptive statistics of the studied sample are shown in Table 1. The mean age at the time of presentation was $12.9 \pm 2.7$ years. The male-to-female ratio was 1:1. The frequency of left convex curves was $26.1 \%$, with no significant difference across the three groups $(p=0.62)$.

The Cobb angle was significantly lower in patients with congenital scoliosis and syringomyelia, as compared to those with CM1 and IS $(p<0.05)$. The frequency of presumed idiopathic scoliosis with intraspinal abnormality was $7.8 \%$; of patients with this anomaly, $48 \%$ had CM1 with syringomyelia and $52 \%$ had IS. The mean length of the syrinx was $7.2 \pm 4.9$ vertebral levels, and the mean SCR was $0.39 \pm 0.2$. The mean length of the syrinx was significantly higher in patients with CM1 and IS, compared to patients with congenital scoliosis $(p<0.05)$, with no significant difference in length between patients with CM1 and IS. The mean SCR was significantly higher in patients with CM1 $(p<0.05)$; however, there was no significant difference in SCR between patients with congenital scoliosis and IS.

The details of the side and severity of syringomyelia are shown in Table 2 . The syrinxes were centrally located in $63.6 \%$ of cases, were to the right in $20.5 \%$, and were to the left in $15.9 \%$. The majority of the syrinxes were medium $(40.9 \%)$ in size, and there were equal numbers of small
Table 2. Frequency of side and severity of syringomyelia in the studied sample

\begin{tabular}{|c|c|c|c|}
\hline Variable & $\begin{array}{c}\text { Chiari } \\
\text { malformation } \\
\text { type } 1(n=12)\end{array}$ & $\begin{array}{c}\text { Idiopathic } \\
\text { syringomyelia } \\
(\mathrm{n}=13)\end{array}$ & $\begin{array}{l}\text { Syringomyelia } \\
\text { in congenital } \\
\text { scoliosis }(n=19)\end{array}$ \\
\hline \multicolumn{4}{|c|}{ Severity of syringomyelia } \\
\hline Small $(n=13)$ & 3 & 2 & 8 \\
\hline Medium ( $n=18$ ) & 4 & 6 & 8 \\
\hline Large $(n=13)$ & 5 & 5 & 3 \\
\hline \multicolumn{4}{|l|}{ Location of syrinx } \\
\hline Right (n=9) & 2 & 3 & 4 \\
\hline Central $(n=28)$ & 9 & 7 & 12 \\
\hline Left $(n=7)$ & 1 & 3 & 3 \\
\hline
\end{tabular}

and large syrinxes (29.5\%). The details of syringomyelia in congenital scoliosis are shown in Table 3. The frequency of syringomyelia in congenital scoliosis was $9.1 \%$. It was seen most frequently in patients with a failure of formation, and $63.2 \%$ had concomitant cord anomalies. The majority of the syrinxes were small to medium in size in patients with congenital scoliosis, whereas the majority of the syrinxes were medium to large in size in patients with CM1 and IS.

\section{Discussion}

The association between syringomyelia and scoliosis is well documented. Rodriguez et al. [11] found the incidence of scoliosis to be $49.1 \%$ in patients with IS. The frequency of scoliosis in patients with CM1 and syringomyelia is as high as $80 \%$ [20]. Some patients who present as adolescents with idiopathic scoliosis may have underlying neuroaxial abnormalities. In a meta-analysis, Faloon et al. [5] found that $8 \%$ of patients presumed to have idiopathic 
Table 3. Details of syringomyelia in congenital scoliosis

\begin{tabular}{|c|c|c|c|c|}
\hline Cord anomaly/vertebral anomaly & Failure of formation ( $n=12$ ) & Failure of segmentation $(n=5)$ & Mixed vertebral anomalies ( $n=2)$ & Total $(n=19)$ \\
\hline \multicolumn{5}{|l|}{ Single cord anomaly $(n=7)$} \\
\hline Syringomyelia & 4 & 3 & 0 & 7 \\
\hline \multicolumn{5}{|l|}{ Multiple cord anomalies ( $\mathrm{n=12)}$} \\
\hline SCM + syringomyelia & 3 & 1 & 1 & 5 \\
\hline Syrinx + tethered cord & 4 & 0 & 0 & 4 \\
\hline SCM + syrinx+tethered cord & 1 & 1 & 1 & 3 \\
\hline
\end{tabular}

SCM, split cord malformation.

scoliosis had intraspinal abnormalities; of these, $35 \%$ had IS and $28 \%$ had CM1 with syringomyelia. In the current study, the frequency of intraspinal abnormalities in patients with presumed idiopathic scoliosis was 7.8\%; of these patients, $48 \%$ had CM1 with syringomyelia and 52\% had IS.

The presence of atypical curve features, such as a left thoracic curve, high thoracic apex, and apical hyperkyphosis, is considered to be pathognomonic of underlying spinal cord abnormalities in patients presumed to have idiopathic scoliosis. Prior reports found the frequencies of left thoracic curves and thoracic hyperkyphosis to be $40 \%-50 \%$ and $29 \%-36 \%$, respectively, in these patients $[7,8]$. In the current study, the frequencies of left thoracic curves and thoracic hyperkyphosis were $26.1 \%$ and $56.8 \%$, respectively. Thus, the absence of atypical curve features should not preclude preoperative MRIs in patients presumed to have adolescent idiopathic scoliosis. The presence of tenderness and subtle signs of neurological deficits on clinical examinations should also be taken into consideration $[5,6,8,9,12]$.

Syrinx characteristics have been extensively studied in patients with IS and CM1 with syringomyelia. In these patients, altered cerebrospinal fluid (CSF) flow at the craniocervical junction, with or without caudal migration of the cerebellar tonsils, results in cavitation and the development of a syrinx, usually in the cervical spine. Some authors found the length of the syrinx, syrinx diameter, and SCR to be significantly larger in patients with CM1 with syringomyelia, compared to those with IS [8]. In the current study, the mean lengths of the syrinx were similar in these two groups. However, the SCR was significantly higher in patients with CM1 with syringomyelia, compared to those with IS. This is similar to the findings of Zhu et al. [8].

Some authors found that the radiological character- istics of scoliosis in both the coronal and sagittal planes were similar in patients with IS and CM1, suggesting the presence of a common pathomechanism. In IS and CM1 patients, the thoracic curves tended to be to the convex side of a centrally located syrinx, which supports the role of spinal cord dysfunction in the pathogenesis of scoliosis $[7,8]$. However, Tan et al. [12] reported no correlation between the radiological features of scoliosis and MRI characteristics of the syrinx, suggesting the presence of other mechanisms. In the current study, the majority of the syrinxes were centrally located, with no discernable pattern to the side of scoliosis and the SDR.

In congenital scoliosis, the deformity progression depends upon the underlying vertebral abnormality, the location of the abnormality, and the age of patient, and is independent of the intraspinal anomaly $[2,13,16]$. In the current series, syrinxes were smaller in size and length in patients with congenital scoliosis, compared to those with IS and CM1 with syringomyelia. In patients with congenital scoliosis, $15.6 \%$ of the syrinxes were large in size, whereas in patients with IS and CM1 with syringomyelia $41.7 \%$ and $38.5 \%$ were large in size, respectively. This result is similar to the findings of Özerdemoglu et al. [14] and Prahinski et al. [21]. The majority of the syrinxes were present in the thoracic spine in cases of congenital scoliosis; in contrast, the majority of the syrinxes were in the cervical spine in cases of IS and CM1. This is probably because patients with congenital scoliosis have indirect tethering at the site of a SCM, a tethered cord, or vertebral anomalies that break down the lining membrane of the central canal, resulting in cavitation and the development of a syrinx. Thus, surgical treatment of the syrinx is not necessary in patients with congenital scoliosis, unless the syrinx is associated with a Chiari malformation or neurological deficit. However, concomitant cord anomalies have to be addressed prior to deformity correction $[4,15]$. 
Syringomyelia in scoliosis can be successfully managed nonoperatively. Asymptomatic patients with a small syrinx and a small curve are unlikely to benefit from posterior fossa decompression and can be closely followed-up to monitor the curve progression and look for neurological deficits $[9,10,20,22]$. In patients with CM1 with syringomyelia and scoliosis, posterior fossa decompression should be done prior to any deformity correction [10]. Historically, shunting procedures in patients with IS have been directly aimed at decompression of the syrinx; however, in recent times these procedures have been less common due to the high risk of neurological complications. In the presence of a significant spinal deformity and a syrinx, a phase-contrast MRI can be used to ascertain CSF flow dynamics at the craniocervical junction and help guide the decision regarding posterior fossa decompression. If the MRI does not show a block in the CSF flow dynamics at the craniocervical junction, deformity correction can safely be performed. Some authors believe posterior fossa decompression should be reserved for those in whom the MRI shows a block at the craniocervical junction. There is no consensus on the rate of curve resolution in patients following posterior fossa decompression; thus, all patients must be closely observed for progression of the deformity until skeletal maturity [22-25].

The strengths of the current study are the inclusion and detailed analysis of consecutive patients with scoliosis associated with syringomyelia at a single center. The drawbacks of the study include its retrospective nature. Due to the small sample, the subgroup analysis carried out in the paper may not reflect any true differences between syringomyelia in patients with CM1, IS, and congenital scoliosis. Another drawback of the study is the lack of a clinical correlation between syrinx characteristics and neurological deficits. Further studies with larger sample sizes may help improve our understanding of syringomyelia in scoliosis.

\section{Conclusions}

Intraspinal anomalies can coexist in patients presumed to have idiopathic scoliosis, even without classical radiological findings. Syrinxes were smaller in size and length and showed distinct differences in patients with congenital scoliosis as compared to patients with IS and CM1, probably due to a different pathomechanism; a majority of syrinxes are associated with concomitant cord anomalies.

\section{Conflict of Interest}

No potential conflict of interest relevant to this article was reported.

\section{References}

1. Kahn EN, Muraszko KM, Maher CO. Prevalence of Chiari I malformation and syringomyelia. Neurosurg Clin N Am 2015;26:501-7.

2. Shen J, Wang Z, Liu J, Xue X, Qiu G. Abnormalities associated with congenital scoliosis: a retrospective study of 226 Chinese surgical cases. Spine (Phila Pa 1976) 2013;38:814-8.

3. Ghandhari H, Tari HV, Ameri E, Safari MB, Fouladi DF. Vertebral, rib, and intraspinal anomalies in congenital scoliosis: a study on 202 Caucasians. Eur Spine J 2015;24:1510-21.

4. Feng F, Tan H, Li X, et al. Radiographic characteristics in congenital scoliosis associated with split cord malformation: a retrospective study of 266 surgical cases. BMC Musculoskelet Disord 2017;18:420.

5. Faloon M, Sahai N, Pierce TP, et al. Incidence of neuraxial abnormalities is approximately $8 \%$ among patients with adolescent idiopathic scoliosis: a metaanalysis. Clin Orthop Relat Res 2018;476:1506-13.

6. Davids JR, Chamberlin E, Blackhurst DW. Indications for magnetic resonance imaging in presumed adolescent idiopathic scoliosis. J Bone Joint Surg Am 2004;86:2187-95.

7. Yeom JS, Lee CK, Park KW, et al. Scoliosis associated with syringomyelia: analysis of MRI and curve progression. Eur Spine J 2007;16:1629-35.

8. Zhu Z, Sha S, Chu WC, et al. Comparison of the scoliosis curve patterns and MRI syrinx cord characteristics of idiopathic syringomyelia versus Chiari I malformation. Eur Spine J 2016;25:517-25.

9. Godzik J, Dardas A, Kelly MP, et al. Comparison of spinal deformity in children with Chiari I malformation with and without syringomyelia: matched cohort study. Eur Spine J 2016;25:619-26.

10. Strahle J, Smith BW, Martinez M, et al. The association between Chiari malformation type I, spinal syrinx, and scoliosis. J Neurosurg Pediatr 2015;15:60711.

11. Rodriguez A, Kuhn EN, Somasundaram A, Couture DE. Management of idiopathic pediatric syringohy- 
dromyelia. J Neurosurg Pediatr 2015;16:452-7.

12. Tan H, Shen J, Feng F, et al. Clinical manifestations and radiological characteristics in patients with idiopathic syringomyelia and scoliosis. Eur Spine J 2018;27:2148-55.

13. McMaster MJ, Ohtsuka K. The natural history of congenital scoliosis: a study of two hundred and fiftyone patients. J Bone Joint Surg Am 1982;64:1128-47.

14. Ozerdemoglu RA, Denis F, Transfeldt EE. Scoliosis associated with syringomyelia: clinical and radiologic correlation. Spine (Phila Pa 1976) 2003;28:1410-7.

15. Ozerdemoglu RA, Transfeldt EE, Denis F. Value of treating primary causes of syrinx in scoliosis associated with syringomyelia. Spine (Phila Pa 1976) 2003;28:806-14.

16. Hedequist D, Emans J. Congenital scoliosis. J Am Acad Orthop Surg 2004;12:266-75.

17. Cobb JR. Outline for the study of scoliosis. Instr Course Lect 1948;5:261-75.

18. Upadhyay SS, Saji MJ, Sell P, Sell B, Yau AC. Longitudinal changes in spinal deformity after anterior spinal surgery for tuberculosis of the spine in adults: a comparative analysis between radical and debridement surgery. Spine (Phila Pa 1976) 1994;19:542-9.

19. Tokunaga M, Minami S, Isobe K, Moriya H, Kitahara H, Nakata Y. Natural history of scoliosis in children with syringomyelia. J Bone Joint Surg Br 2001;83:3716.
20. Wang G, Sun J, Jiang Z, Cui X, Cui J. One-stage correction surgery of scoliosis associated with syringomyelia: is it safe to leave untreated a syrinx without neurological symptom? J Spinal Disord Tech 2015;28:E260-4.

21. Prahinski JR, Polly DW Jr, McHale KA, Ellenbogen RG. Occult intraspinal anomalies in congenital scoliosis. J Pediatr Orthop 2000;20:59-63.

22. Roy AK, Slimack NP, Ganju A. Idiopathic syringomyelia: retrospective case series, comprehensive review, and update on management. Neurosurg Focus 2011;31:E15.

23. Sha S, Qiu Y, Sun W, Han X, Zhu W, Zhu Z. Does surgical correction of right thoracic scoliosis in syringomyelia produce outcomes similar to those in adolescent idiopathic scoliosis? J Bone Joint Surg Am 2016;98:295-302.

24. Struck AF, Haughton VM. Idiopathic syringomyelia: phase-contrast MR of cerebrospinal fluid flow dynamics at level of foramen magnum. Radiology 2009;253:184-90.

25. Chotai S, Basem J, Gannon S, et al. Effect of posterior fossa decompression for Chiari malformation-I on scoliosis. Pediatr Neurosurg 2018;53:108-15. 